\title{
Punishment promotes response control deficits in obsessive-compulsive disorder: evidence from a motivational go/no-go task
}

\author{
S. Morein-Zamir*, M. Papmeyer, C. M. Gillan, M. J. Crockett, N. A. Fineberg, B. J. Sahakian \\ and T. W. Robbins \\ MRC/Wellcome Trust Behavioural and Clinical Neuroscience Institute (BCNI), Department of Experimental Psychology, University of \\ Cambridge, Cambridge, UK
}

Background. Obsessive-compulsive disorder (OCD) has been associated with response inhibition deficits under motivationally neutral contingencies. We examined response inhibition performance in the presence of reward and punishment. We further investigated whether the hypothesized difficulties in flexibly updating behaviour based on external feedback in OCD would also lead to a reduced ability to adjust to changes in the reward and punishment contingencies.

Method. Participants completed a go/no-go task that used punishments or rewards to promote response activation or suppression. The task was administered to OCD patients free of current Axis-I co-morbidities including major depression $(n=20)$ and a group of healthy controls $(n=32)$.

Results. Compared with controls, patients with OCD had increased commission errors in punishment conditions, and failed to slow down immediately after receiving punishment. The punishment-induced increase in commission errors correlated with self-report measures of OCD symptom severity. Additionally, patients did not differ from controls in adapting their overall response style to the changes in task contingencies.

Conclusions. Individuals with OCD showed reduced response control selectively under punishment conditions, manifesting in an impulsive response style that was related to their current symptom severity. This stresses failures of cognitive control in OCD, particularly under negative motivational contingencies.

Received 6 December 2011; Revised 5 April 2012; Accepted 10 April 2012 ; First published online 14 May 2012

Key words: Inhibition, obsessive-compulsive disorder, punishment, response control, reward.

\section{Introduction}

Obsessive-compulsive disorder (OCD) is characterized by intrusive thoughts (obsessions) and/or repetitive behaviours (compulsions). Neurobiological models of OCD stress the dysfunctional role of frontostriatal circuits (Graybiel \& Rauch, 2000; Menzies et al. 2008). While the orbitofronto-striatal circuit has received particular attention, abnormalities are present in additional frontal regions such as the dorsolateral prefrontal cortex and inferior frontal gyrus (van den Heuvel et al. 2005; Menzies et al. 2007). Given the abnormalities in fronto-striatal circuitry, executive functions have been of interest in OCD. While impairments are not widespread, deficits have been reported in inhibitory control of responses and cognitive flexibility

\footnotetext{
* Address for correspondence: S. Morein-Zamir, Ph.D., MRC/ Wellcome Trust Behavioural and Clinical Neuroscience Institute (BCNI), Department of Experimental Psychology, University of Cambridge, Downing Site, Cambridge CB2 3EB, UK.

(Email: sm658@cam.ac.uk)
}

and their neural correlates (Chamberlain et al. 2007b, 2008; Menzies et al. 2007). Difficulties in suppressing inappropriate actions may be linked to the repetitive nature of OCD symptoms. Similarly, cognitive inflexibility may be related to problems in adjusting to changing internal and external requirements. Motor inhibition deficits and cognitive inflexibility have also been suggested as putative endophenotypes, with impairments observed in first-degree relatives of OCD patients (Chamberlain et al. 2007b).

Response inhibition impairments in OCD have been found using go/no-go, stop signal and anti-saccade tasks (Menzies et al. 2008). For example, OCD patients had slowed stop-signal response times (RTs) despite response latencies that were comparable with those of healthy controls (Chamberlain et al. 2007b; MoreinZamir et al. 2010). Findings from go/no-go tasks have been more varied, though neural abnormalities are often apparent in frontal and striatal substrates (Maltby et al. 2005; Roth et al. 2007; Page et al. 2009). In this task, participants perform speeded responses to

The online version of this article is published within an Open Access environment subject to the conditions of the Creative Commons Attribution-NonCommercial-ShareAlike licence <http://creativecommons.org/licenses/by-nc-sa/2.5/ $>$. The written permission of Cambridge University Press must be obtained for commercial re-use. 
target stimuli, and withhold these motor responses when non-target stimuli are presented. Some studies have shown OCD patients to have difficulties withholding responses, resulting in increased commission errors (Bannon et al. 2002; Penades et al. 2007). Others found unimpaired performance (Maltby et al. 2005; Bohne et al. 2008; Page et al. 2009) while still others have indicated difficulties only when introducing additional cognitive demands such as reversing the go/no-go rules (Watkins et al. 2005).

Response inhibition performance in OCD is typically monitored in motivationally neutral situations. Given orbitofronto-striatal involvement in OCD and its importance in processing reward and punishment (O'Doherty, 2007), we examined whether inhibitory control in OCD is modulated by positive and negative feedback. There is evidence to suggest that learning based on negative feedback is abnormal in OCD, with patients having difficulties learning from punishing feedback even after extensive practice (Nielen et al. 2009). Additionally, while OCD patients could learn using positive and negative feedback as well as controls in a different task, they later demonstrated an enhanced negative learning bias (Endrass et al. 2011). Thus, the patients learned to avoid choosing the stimulus initially associated with negative feedback better than they had learned to choose the stimulus initially associated with positive feedback. Abnormal neural responses to gain and loss incentives have also been reported, although results are presently largely inconsistent as to whether abnormalities are specific to gain or loss, and whether they occur when receiving rather than anticipating the feedback (Remijnse et al. 2006; Figee et al. 2011; Jung et al. 2011; Stern et al. 2011).

Positive and negative feedback is also important in modulating behavioural flexibility. Cognitive flexibility involves effective behavioural adaptation to a changing environment and is mediated by frontostriatal loops including the orbitofrontal, the ventrolateral prefrontal cortex and the striatum (Kehagia et al. 2010). In OCD, beliefs and behaviours are often resistant to change, despite their negative consequences. In cued switching tasks in the absence of feedback, OCD patients do not demonstrate increased switch costs that would be indicative of reduced flexibility (Moritz et al. 2004; Gu et al. 2008). Cognitive inflexibility in OCD patients has been reported when they are required to learn new rules based on feedback; specifically, impairments in medicated patients were found in an attentional set-shifting task (Watkins et al. 2005; Chamberlain et al. 2007b), although evidence from the Wisconsin Card Sorting Task has been inconsistent (Kuelz et al. 2004). Adjusting behaviour flexibly is also necessary when responses formerly associated with positive consequences are now associated with negative consequences. Although performance on reversal learning tasks can be intact in OCD (Chamberlain et al. 2007a), neural abnormalities in regions including the orbitofrontal cortex have at times but not always been reported (Remijnse et al. 2006; Chamberlain et al. 2008; though see also Ersche et al. 2011). Reduced sensitivity to the outcome of actions (Gillan et al. 2011) may further promote decreased flexibility in a changing environment, with a deficit in the processing of action-related feedback resulting in difficulties in updating action goals (Olley et al. 2007; Nielen et al. 2009). Thus, at present it remains unclear which particular aspects of cognitive flexibility are impaired in OCD.

In the current study, we examined go/no-go performance in OCD under conditions of multimodal reward or punishment (Crockett et al. 2009). Performance was punished or rewarded at different times with feedback involving the loss or gain of points as well as sounds and social cues. It was hypothesized that OCD patients would show impairments particularly under punishment conditions. Further, the feedback was used to induce a general propensity towards responding (i.e. a bias to go) or towards suppressing responses (i.e. a bias to not go), so that response style must be adapted to the changing situational context. Thus, large rewards for correct go responses should promote a stronger propensity to respond than to withhold responses. Large punishments for incorrectly withholding responses on go trials (omission errors) should also promote a tendency to respond. Conversely, large rewards for correctly withholding responses on no-go trials and large punishments for incorrectly responding on no-go trials (commission errors) should both promote an increased propensity to suppress responding. Flexibility would manifest in appropriately adjusting behaviour to the changes in contingencies, such that under a bias to activate responding there would be a greater number of responses and faster responding. Under a bias to suppress responding there would be fewer responses and responding would be slower. Inflexibility would manifest in smaller differences between conditions promoting response activation versus suppression.

\section{Method \\ Participants}

A total of 20 OCD patients (55\% female) were recruited from a specialist OCD out-patient clinic following screening by a consultant psychiatrist (N.A.F.) to ensure that they met Diagnostic and Statistical Manual of Mental Disorders, 4th edn, text revision (DSM-IV-TR; APA, 2000) criteria for OCD and were 
Table 1. Demographic and clinical characteristics of the OCD patient and control groups

\begin{tabular}{lrrrrrc}
\hline & \multicolumn{2}{l}{ OCD patients $(n=20)$} & \multicolumn{1}{c}{ Controls $(n=32)$} & $Z$ & $p$ \\
\hline Age, years & 44.55 & $(8.42)$ & 41.84 & $(13.00)$ & 0.79 & $>0.43$ \\
Verbal IQ & 115.95 & $(7.86)$ & 117.48 & $(6.88)$ & 0.76 & $>0.44$ \\
Length of & 15.45 & $(2.68)$ & 16.04 & $(2.30)$ & 0.75 & $>0.45$ \\
$\quad$ education, years & & & & & & \\
MADRS & 5.60 & $(4.08)$ & & & & \\
YBOCS & 19.80 & $(5.13)$ & & & & \\
OCI-R & 28.55 & $(12.91)$ & 8.26 & $(5.30)$ & 5.24 & $<0.001$ \\
PI-R & 42.50 & $(23.10)$ & 12.77 & $(13.48)$ & 4.67 & $<0.001$ \\
STAI-S & 42.00 & $(12.99)$ & 30.35 & $(6.20)$ & 3.42 & $<0.001$ \\
STAI-T & 56.00 & $(11.65)$ & 35.97 & $(8.29)$ & 5.12 & $<0.001$ \\
& & & & & & \\
\hline
\end{tabular}

OCD, Obsessive-compulsive disorder; IQ, intelligence quotient; MADRS,

Montromery-Asberg Depression Rating Scale; YBOCS, Yale-Brown Obsessive

Compulsive Scale; OCI-R, Obsessive Compulsive Inventory - Revised; PI-R, Padua

Inventory - Revised; STAI-S, State-Trait Anxiety Inventory, state; STAI-T, State-Trait

Anxiety Inventory, trait.

Data are given as mean (standard deviation).

free of current co-morbid Axis-I disorders, including major depression. Screening upon entry to the clinic was assessed using an extended semi-structured clinical interview, which screened for affective disorders including bipolar disorder, schizophrenia, anxiety disorders, somatoform disorders, OCD spectrum disorders including tic and Tourette's disorder, body dysmorphic disorder, trichotillomania, compulsive skin-picking, pathological gambling, hoarding, autistic spectrum disorders, attention deficit hyperactivity disorder and substance-use disorders. Subjects were subsequently monitored by the consultant psychiatrist on a regular basis to track ongoing symptoms and the emergence of any new ones. All but one of the patients were receiving selective serotonin reuptake inhibitors (SSRIs): seven with fluoxetine, $40-100 \mathrm{mg}$; five with escitalopram, 10-30 mg; two with fluvoxamine, 100-150 mg; two with sertraline, $200 \mathrm{mg}$; two with paroxetine, 40-60 mg; and one with citalopram, $60 \mathrm{mg}$; one patient was also on clomipramine $(25 \mathrm{mg})$ and two were also on antipsychotics (quetiapine $100 \mathrm{mg}$ and flupenthixol $0.5 \mathrm{mg}$ ).

A group of 32 controls (59\% female), reporting no psychiatric illness, history of head injury or neurological disorder or psychotropic medication use, also completed the task. All participants were fluent English speakers/readers, though three of the controls were not native English speakers. All but two of the control group and one patient reported being right handed. Group characteristics are presented in Table 1. OCD symptom severity was assessed by the Yale-Brown Obsessive Compulsive Scale (YBOCS; Goodman et al. 1989), the Obsessive Compulsive Inventory - Revised (OCI-R; Foa et al. 2002) and the
Padua Inventory - Revised (PI-R; Burns et al. 1996). Anxiety was assessed with the State-Trait Anxiety Inventory (STAI; Spielberger et al. 1983), depression severity with the Montgomery-Asberg Depression Rating Scale (Montgomery \& Asberg, 1979), and verbal intelligence with the National Adult Reading Test (Nelson, 1982). Participants completed two other behavioural tasks, unrelated to the present study (Gillan et al. 2011), in a counterbalanced order during the same session. All tasks and questionnaires were administered by a graduate psychologist trained in testing OCD patients. The study was approved by the Hertfordshire Research Ethics Committee, and written informed consent was obtained from all participants before beginning the session.

\section{Procedure}

Participants performed a go/no-go task and completed four experimental feedback blocks in counterbalanced order (Crockett et al. 2009). Feedback blocks used reward or punishment to create either respond (go) or inhibit (no-go) biases. In the go bias via reward block, correct responses (hits) were rewarded, whereby correct go responses yielded large rewards and correct no-go responses yielded small rewards. In the no-go bias via reward block, correct no-go responses yielded large rewards and correct go responses yielded small rewards. In both reward blocks, mistakes resulted in neutral feedback. Small rewards earned one point, a high tone and a mildly happy face; large rewards earned 10 points, a flourishing tone and an extremely happy face. In the go bias via punishment block, incorrect responses were punished, whereby 


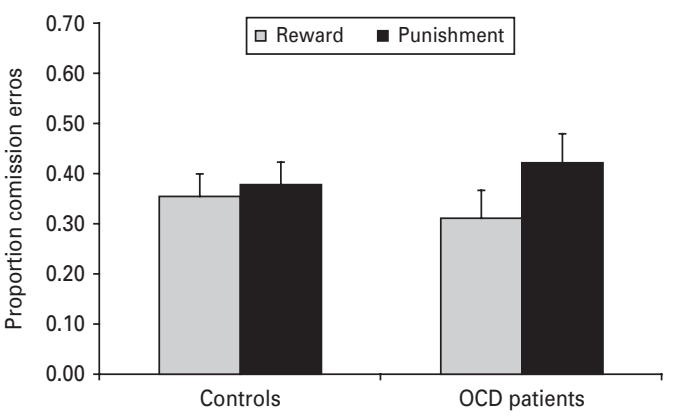

Fig. 1. Proportion of commission errors in controls and obsessive-compulsive disorder (OCD) patients under conditions of reward and punishment. Values are means, with standard errors represented by vertical bars.

incorrect go responses (commission errors) resulted in small punishment and incorrect no-go responses resulted in large punishment. In the no-go bias via punishment block, incorrect no-go responses resulted in small punishment and incorrect go responses resulted in large punishment. In both punishment blocks, correct responses resulted in neutral feedback. Small punishments lost one point, resulted in a short buzzing tone and a mildly angry face while large punishments lost 10 points, resulted in a long buzzing tone and an extremely angry face. All feedback was presented for $1500 \mathrm{~ms}$.

In each block of 36 trials, half were go and half were no-go trials. Participants responded to blue and yellow checkerboard stimuli by pressing a button if the target colour was in the majority and did not respond if it was in the minority, with target colour counterbalanced across participants. Blue-to-yellow square frequency established easy (e.g. 16 yellow and nine blue) versus difficult (e.g. 13 yellow and 12 blue) stimuli, with difficulty counterbalanced between go and no-go trials. The task began with 48 practice trials without feedback to minimize learning effects. Stimuli were presented for $2000 \mathrm{~ms}$, with an inter-trial interval of $1500 \mathrm{~ms}$. Mean RT for correct responses in the practice session constituted the individual's stimulus duration, to match task difficulty across participants. Feedback blocks were interspersed among nofeedback blocks, to allow response tendencies to return to baseline, totalling nine blocks. Each block began with four guided practice trials to learn the upcoming response contingency.

\section{Data analysis}

Commission errors and hits were computed for each block. As performance neared a ceiling on easy trials, these calculations were restricted to difficult trials. Mean RTs for correct responses were computed across all trials. To investigate the immediate impact of punishment on responding, RTs were analysed in punishment blocks on trials after punishment versus trials after non-punishment. Proportion data were arcsine transformed and RT data logarithm transformed, though untransformed means are shown throughout. Repeated-measures analyses of variance were conducted with group as the between-subjects factor and response style (go versus no-go bias) and feedback (reward versus punishment) as within-subjects factors. Secondary analyses examined performance in the nofeedback blocks to verify whether there were any lasting effects. Pearson correlation coefficients were computed between clinical characteristics of OCD patients and task performance indices. Non-parametric Mann-Whitney $U$ tests were used when comparing clinical characteristics. All comparisons were twotailed with an $\alpha$ of 0.05 .

\section{Results}

\section{Clinical and psychological rating scales}

OCD patient and controls were matched for gender distribution $[\chi(1)=0.10, p>0.75]$, dominant hand $[\chi(1)=0.04, p>0.85]$, age, years of education and verbal intelligence (see Table 1). Patients had worse OCD symptoms (OCI-R and PI-R) and increased anxiety (STAI).

\section{Commission errors}

Analyses of commission errors as a measure of response inhibition revealed more commission errors in punishment blocks [main effect of feedback: $F(1,50)=9.09, p<0.01]$. As seen in Fig. 1, this was moderated by an interaction between group and feedback $[F(1,50)=4.77, p<0.05]$, whereby feedback influenced commission errors in the OCD patients $[F(1,50)=10.97, p<0.01]$ but not in controls $[F(1,50)$ $<1, p>0.50]$. A direct comparison between the groups of the difference scores between reward and punishment for each participant was significant $[F(1,50)=$ $4.23, p<0.05]$, with a Cohen's $d$ effect size of 0.59 . Additionally, there was an interaction between response style and feedback $[F(1,50)=4.57, p<0.05]$, with an effect of feedback in no-go biased blocks $[F(1,50)=11.21, p<0.01]$, but not go biased blocks $[F(1,50)<1, p>0.88]$. There was no significant interaction between group, response style and feedback $[F(1,50)<1, p>0.80]$.

\section{Correct go responses}

The proportion of hits was greater in go bias compared with no-go bias, with participants more likely to 

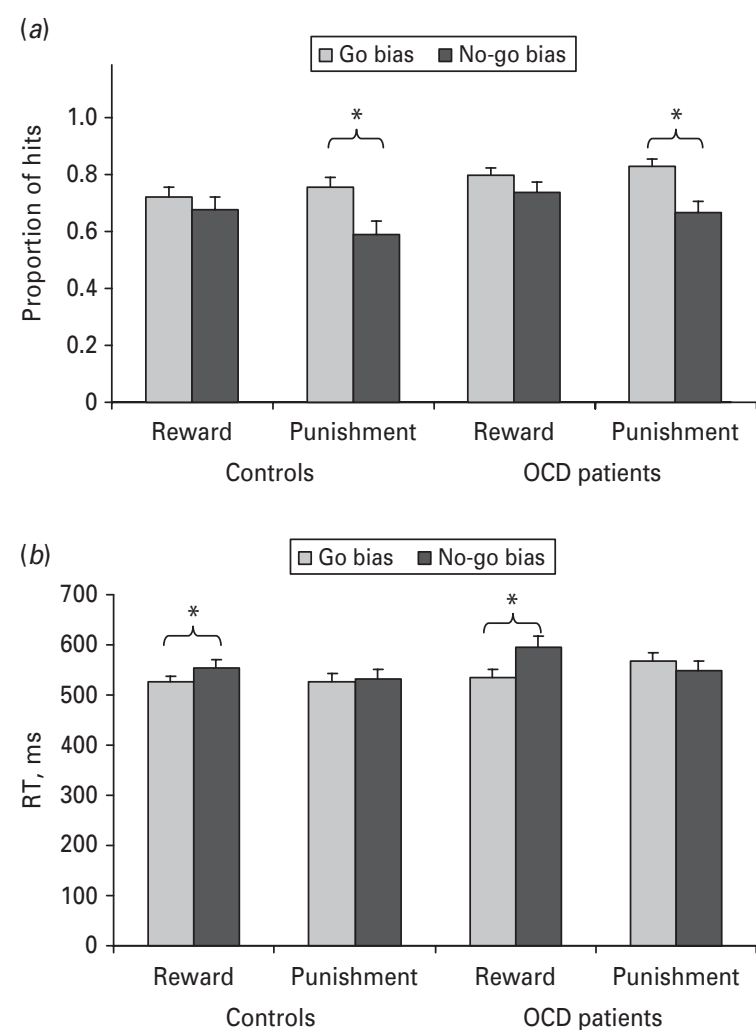

Fig. 2. (a) Hits for controls and obsessive-compulsive disorder (OCD) patients for go and no-go biases under conditions of reward and punishment. (b) Response times (RTs) for controls and patients for go and no-go biases under conditions of reward and punishment. Values are means, with standard errors represented by vertical bars. * Mean value was significantly different from that for the no-go bias condition $(p<0.05)$.

respond in the go bias conditions (0.78 v. 0.67, respectively) $[F(1,50)=19.19, p<0.001]$. There was also an interaction between feedback and response style $[F(1,50)=4.90, p<0.05]$, such that there was a significant response style effect under punishment $[F(1,50)=21.20, p<0.001]$, but not under reward $[F(1,50)=1.45, p>0.22]$. The three-way interaction between group, feedback and response style was not significant $[F(1,50)<1, p>0.50]$. However, we examined the effect of response style under punishment for each group separately to ascertain whether both groups adjusted their responding. As seen in Fig. $2 a$, both controls $[F(1,50)=14.29, p<0.01]$ and OCD patients $[F(1,50)=8.30, p<0.01]$ adjusted their responses under punishment with reduced responding under the no-go bias.

An analysis of correct RTs showed faster responses under go compared with no-go bias (539 v. $558 \mathrm{ms,}$ respectively) $[F(1,50)=8.82, p<0.05]$. There was an interaction between response style and feedback

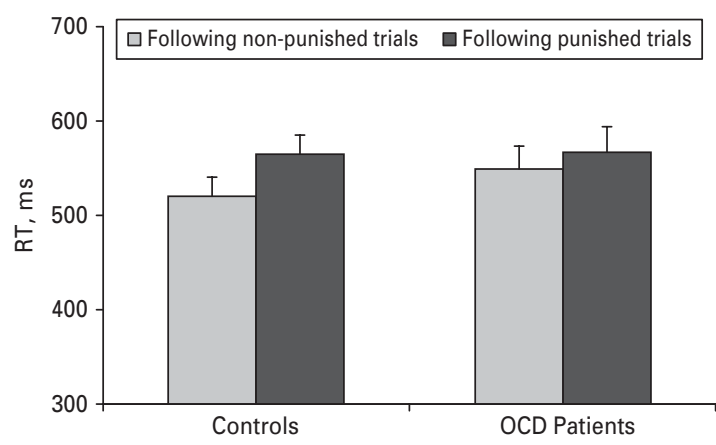

Fig. 3. Response times (RTs) following punished versus non-punished trials in controls and obsessive-compulsive disorder (OCD) patients. Values are means, with standard errors represented by vertical bars.

$[F(1,50)=22.07, p<0.01]$, such that latencies were faster in go compared with no-go bias conditions under reward $[F(1,50)=21.69, p<0.001]$ but not punishment $[F(1,50)=1.06, p>0.30]$. Finally, a three-way interaction between group, response style and feedback $[F(1,50)=6.13, p<0.05]$ appeared to stem from marginally faster RTs in no-go compared with the go bias punishment blocks for patients $[F(1,50)=3.20$, $p<0.08]$ but not controls $[F(1,50)<1, p>0.54]$. Importantly, the effect of response style found under reward was significant both for the controls $[F(1,50)=$ 6.67, $p<0.05]$ and the patients $[F(1,50)=16.91$, $p<0.01]$, indicating that OCD patients adjusted their responses similarly to controls (see Fig. $2 b$ ).

\section{Slowing on trials immediately following punishment}

We further investigated whether patients responded differently from controls on trials immediately following punishment. Latencies in the punished conditions were compared between trials after correct (nonpunished) versus after incorrect responses (punished). There was a general slowing following punishment $[F(1,50)=20.02, p<0.001]$. However, as seen in Fig. 3, this was moderated by an interaction between group and prior punishment $[F(1,50)=4.49, p<0.05]$ whereby controls responded slower after punishment $[F(1,50)=28.26, \quad p<0.001]$, but patients did not $[F(1,50)=2.26, p>0.13]$. A direct comparison between the groups of the difference scores between postpunished and post-non-punished trials was significant $[F(1,50)=4.49, p<0.05]$, with a Cohen's $d$ effect size of 0.60 .

\section{Additional analyses}

Analyses of the no-feedback blocks did not reveal any effects of previous feedback, response style or group. 

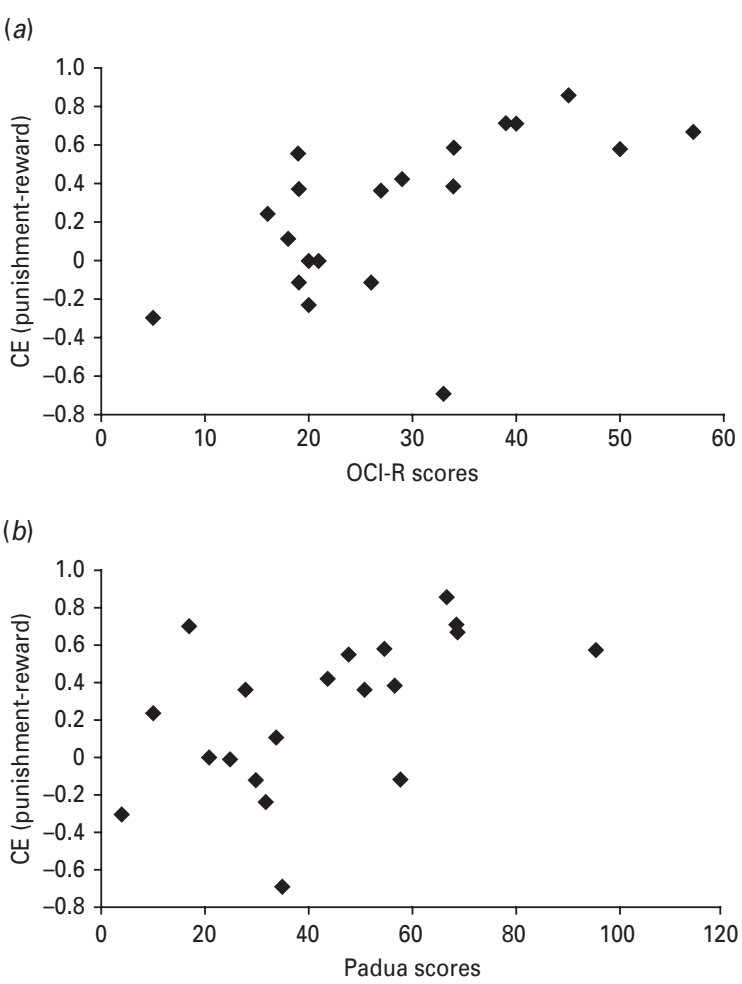

Fig. 4. (a) Scatterplot of increased commission errors (CE) under punishment and scores on the Obsessive Compulsive Inventory - Revised (OCI-R) in the patient group (Pearson correlation of 0.60). (b) Scatterplot of increased commission errors under punishment and scores on the Padua Inventory - Revised (PI-R) (Pearson correlation of 0.53).

To examine whether slowing on trials immediately following punishment was specific to punishment rather than error, a similar analysis was conducted in the no-feedback blocks. There was significant slowing after an incorrect trial $[F(1,49)=4.79, p<0.05]$, but no interaction with group $[F(1,49)<1, p>0.43]$. We examined correlations between patient characteristics and task-related measures. Punishment-related increase in commission errors correlated significantly with both OCI-R and PI-R but not YBOCS scores $[r=0.60, \quad t(18)=3.19, p<0.01 ; r=0.53, t(18)=2.64$, $p<0.017$; and $r=0.29, \quad t(18)=0.86, \quad p>0.40$, respectively], such that worse self-reported OCD symptoms were associated with increased punishment-related commission errors (see Fig. 4). These correlations remained significant when partialling out anxiety and depression ratings [partial $r^{\prime} s=0.71$ and 0.62 for OCI-R and PI-R, respectively]. No other correlations were significant.

To examine a possible role for SSRI medication, SSRI equivalence values were computed for each patient (Koran et al. 2007), as were imipramine equivalences (Bollini et al. 1999). SSRI equivalence values ranged from 0 to 125 (mean=7.25, S.D. $=31.21$ ), and imipramine equivalences ranged from 0 to 500 $($ mean $=229.85$, S.D. $=156.91)$. SSRI equivalence values did not correlate with punishment-induced increase in commission errors $[r=-0.05, t(18)=-0.23, p>0.81]$ nor with post-error slowing $[r=-0.04, t(18)=-0.17$, $p>0.85]$. Similar results were found with imipramine equivalence values.

\section{Discussion}

We examined inhibitory control in non-depressed OCD patients compared with healthy controls under conditions of punishment and reward. Individuals with OCD showed reduced response control manifesting in an impulsive response style selectively under punishment conditions. Specifically, patients had increased commission errors in the punishment blocks overall and did not demonstrate the expected slowing following punishment (Figs 1 and 3). The increase in punishment-related commission errors correlated with two indices of symptom severity (Fig. 4). At the same time, patients adjusted their response style similarly to controls based on the motivational contingencies. Under punishment, both groups responded more in the go compared with the no-go bias conditions (Fig. 2a). Under reward, both groups responded faster in the go compared with the no-go bias conditions (Fig. 2b).

In the present study, response inhibition impairments in OCD were found only under conditions of punishment. Mere negative emotion induced by the punishment is unlikely to explain the present findings, as in previous studies negative affective stimuli did not influence response inhibition in OCD (Chamberlain et al. 2007a; Morein-Zamir et al. 2010). Rather, the presence and anticipation of aversive performance-related punishment probably underlie the current results. Moreover, because task difficulty was individually set, some punishment was unavoidable. Avoidance behaviours are common and inherent to OCD, with patients learning particularly well to avoid negative stimuli (Endrass et al. 2011). Punishmentrelated increases in commission errors correlated with self-reported measures of OCD severity controlling for levels of depression and anxiety, suggesting that it may be associated with OCD rather than depressive symptoms. At the same time, no correlation was observed with YBOCS scores. This disparity may be due to the nature of the measures; the YBOCS is openended, interview-based and includes items regarding the patient's resistance and control over their symptoms, whereas the self-report questionnaires focus on specific possible symptoms (e.g. repeatedly checking doors, windows, drawers, etc.) and patients rate the 
intensity of distress or degree of disturbance. In any case, abnormal response control under punishment is consistent with the notion that OCD patients do not merely have an 'abnormal response to negative feedback' or a negative bias in processing information as found in depression (Sahakian \& Morein-Zamir, 2011). These findings are also unlikely to be attributable to negative urgency (Cyders \& Smith, 2008), whereby individuals tend to engage in rash, illadvised actions when distressed, as other performance indices (e.g. latencies and response style effects) were not abnormal under punishment. Rather, punishment plays a more complex role in modulating inhibitory control difficulties.

Reward does not lead to a normalization of performance in OCD patients, as there was no difference between the groups in the no-feedback blocks. It is likely that in the reward and no-feedback conditions, inhibitory demands were not sufficiently taxing, as no-go trials were frequent, constituting half the trials. Response inhibition difficulties would be most apparent when countermanding an already initiated response (Menzies et al. 2007) or with additional processing demands such as complex or changing response-stimulus mappings (Bannon et al. 2002; Watkins et al. 2005; Nielen et al. 2009). Punishment appears to be another factor that places additional demands leading to reduced inhibitory control in OCD. Inhibitory control impairments particularly under impending punishment may distinguish OCD from other disorders also characterized by inhibitory difficulties, such as drug addiction and attention deficit hyperactivity disorder (e.g. Monterosso et al. 2005; Lipszyc \& Schachar, 2010). Moreover, magnified inhibitory deficits under aversive conditions may be specific to the expression of OCD, given that inhibitory deficits under neutral conditions were also found in non-affected first-degree relatives (Menzies et al. 2007). In support of this, the current inhibitory impairment appeared to be state dependent, in contrast to previous findings where inhibitory performance under neutral conditions did not correlate with clinical characteristics (Chamberlain et al. 2007b). It is also possible that other anxiety disorders will show inhibitory impairments under negative motivational contingencies, despite there being no consistent evidence for impaired inhibition under neutral contingencies (Lipszyc \& Schachar, 2010). Inhibitory control under the threat of punishment is likely to depend both on the functioning of neural substrates mediating response inhibition, such as the inferior frontal gyrus (Menzies et al. 2008), and additional systems activated under aversive conditions, such as affective orbitofrontal-ventral striatal systems (Graybiel \& Rauch, 2000). It may well be that the re- cruitment and interplay of several neural substrates, each thought to be abnormal in OCD (Menzies et al. 2008) preclude the use of potential compensatory neural mechanisms sometimes reported (Rauch et al. 2007; Henseler et al. 2008), and result in behavioural impairments.

Punishment-related failures in response control in the patients were also evidenced by the lack of slowing immediately following punishment. Slowing following errors is typically interpreted as the engagement of cognitive control mechanisms. Seemingly, the results appear inconsistent with reports of an overactive monitoring system in OCD (Gehring et al. 2000; Stern et al. 2011). However, most studies focus on neural correlates at the time of committing an error and do not find behavioural differences in post-error slowing (Endrass et al. 2010). The reduced post-error slowing in OCD here probably results from the presence of punishment, as no group differences were observed in the no-feedback blocks. This is consistent with the finding that neural hyperactivations associated with errors in OCD, in the ventromedial prefrontal cortex and right anterior insula/frontal operculum, are even greater when the errors are associated with monetary loss (Stern et al. 2011).

We also found that OCD patients and controls showed similar levels of adaptability to changing task contingencies, suggesting that the patients were able to flexibly shift between response styles. Despite predicting that cognitive rigidity may manifest in reduced response style effects in patients, this was not the case. Performance in the present study was measured following practice, and the requirement to change response style was explicitly conveyed via instructions. Thus, as is often the case with instructed task switching (Moritz et al. 2004), OCD patients did not appear to be impaired when the task rules were explicit and practised. Abnormalities in the neural substrates mediating effective behavioural flexibility, such as the medial and lateral prefrontal cortices and the basal ganglia (Graybiel \& Rauch, 2000; Kehagia et al. 2010), do not necessarily translate into impaired performance. It may well be that cognitive rigidity in OCD manifests primarily during learning, where there is substantial uncertainty in determining the most appropriate behaviour, and action-related feedback is necessary to determine future performance (Nielen et al. 2009). This study, however, shows that patients can update the balance between opposing action goals, at least when these bear no relation to their personal symptomatology (Olley et al. 2007). With more precise conceptual definitions of cognitive flexibility and cognitive tasks to test its different features, it may be possible in future to better understand the everyday rigidity in behaviour observed in OCD. 
A previous study using a similar task found slowing in the punished relative to rewarded conditions and this was abolished under acute tryptophan depletion (Crockett et al. 2009). In the present study, patients and controls did not respond slower under punishment. However, there are important methodological differences between the studies. Namely, in the current study accuracy was emphasized over speed and performance was not associated with monetary consequences. Additionally, participants were considerably older and performed the task only once. In the present study commission errors were considerably lower than in the previous study, suggesting that participants may have responded more conservatively. That a reduced tendency to respond manifested under punishment in increased misses while under reward it manifested in slower responding is in line with this possibility.

The present study found an inhibitory deficit in OCD specifically under punishment conditions. These are precisely the conditions where serotonin plays a role in behavioural suppression (Cools et al. 2008; Crockett et al. 2009), and tryptophan depletion eliminated punishment-induced inhibition in the same task (Crockett et al. 2009). The OCD patients in the present study were under chronic SSRI medication. However, it is difficult to see how this drug treatment, which enhances serotonergic activity (Stahl, 2008), could have produced the present findings, given the qualitatively similar effects of tryptophan depletion. Moreover, medication dosage did not correlate with task-related performance. It is perhaps more likely that the medication only incompletely ameliorated the disinhibition of responding under punishment. Nevertheless, serotonergic manipulations do not generally influence inhibitory performance under neutral contingencies (Evers et al. 2006), and previous studies did not find that medication influenced reward- and punishment-related error performance in OCD (Nielen et al. 2009; Stern et al. 2010). It is nevertheless possible that unmedicated patients would have additional difficulties in the task, for example, showing a reduced response-style effect. In this study patients were not actively screened for personality disorders or lifetime Axis-I disorders, such as major depressive disorder, and although we excluded Tourette's disorder, tic severity was not systematically evaluated. These factors may have affected patient performance, although it remains unclear how they would be linked to the impairments found in the present study. Future studies may investigate inhibitory control in Axis-II disorders and how it may be modulated under motivational contingencies, as this may provide insight into the results obtained in patients with various AxisI disorders.
In summary, medicated yet symptomatic OCD patients could flexibly adjust their response style under changes in punishment and reward contingencies. At the same time they demonstrated a more impulsive response style compared with controls selectively under punishment conditions. The present study stresses abnormal cognitive control processing, particularly during punishment, in OCD.

\section{Acknowledgements}

The authors thank the volunteers who took part. This research was funded by a Wellcome Trust grant awarded to T.W.R., B. J. Everitt, A. C. Roberts, J. W. Dalley and B.J.S. (no. 089589/Z/09/Z). Work was completed at the Behavioural and Clinical Neuroscience Institute, which is supported by a joint award from the Medical Research Council and Wellcome Trust (no. G00001354). S.M.-Z. is supported by the Wellcome Trust grant above (089589/Z/09/Z). C.M.G. is supported by a studentship from the Medical Research Council. M.J.C. is supported by the Gates Cambridge Trust.

\section{Declaration of Interest}

B.J.S. has consulted for Boehringer-Ingelheim, Cambridge Cognition, Eli Lilly, GlaxoSmithKline, Novartis and Shire; she has received honoraria for Grand Rounds in Psychiatry at Massachusetts General Hospital and for speaking at the International Conference on Cognitive Dysfunction in Schizophrenia and Mood Disorders; she was on the Medical Research Council Neurosciences and Mental Health Board and on the Science Coordination Team for the Foresight Project on Mental Capital and Wellbeing; she has been on Panel LS5 for the European Research Council; and she receives an honorarium from the Journal of Psychological Medicine. She holds a grant funded by Johnson and Johnson. N.A.F. has consulted for Bristol-Myers Squibb, GlaxoSmithKline and Lundbeck and Servier; she has received research support from AstraZeneca, Cephalon, the European College of Neuropsychopharmacology, GlaxoSmithKline, Lundbeck, Servier and Wellcome; she has received honoraria for lectures at scientific meetings from AstraZeneca, Janssen, Jazz, Lundbeck, Servier and Wyeth; and she has received financial support to attend scientific meetings from AstraZeneca, Bristol-Myers Squibb, Cephalon, International College of Obsessive-Compulsive Spectrum Disorders, Janssen, Jazz, Lundbeck, Servier and Wyeth. T.W.R. has consulted for Cambridge Cognition, Lundbeck, Pfizer and Lilly, and has received 
research grants from GlaxoSmithKline, Lundbeck and Lilly.

\section{References}

APA (2000). Diagnostic and Statistical Manual of Mental Disorders (DSM-IV-TR). American Psychiatric Association: Washington, DC.

Bannon S, Gonsalvez CJ, Croft RJ, Boyce PM (2002). Response inhibition deficits in obsessive-compulsive disorder. Psychiatry Research 110, 165-174.

Bohne A, Savage CR, Deckersbach T, Keuthen NJ, Wilhelm S (2008). Motor inhibition in trichotillomania and obsessive-compulsive disorder. Journal of Psychiatric Research 42, 141-150.

Bollini P, Pampallona S, Tibaldi G, Kupelnick B, Munizza C (1999). Effectiveness of antidepressants meta-analysis of dose-effect relationships in randomised clinical trials. British Journal of Psychiatry 174, 297-303.

Burns GL, Keortge SG, Formea GM, Sternberger LG (1996). Revision of the Padua Inventory of Obsessive Compulsive Disorder Symptoms: distinctions between worry, obsessions, and compulsions. Behavioural Research and Therapy 34, 163-173.

Chamberlain SR, Fineberg NA, Blackwell AD, Clark L, Robbins TW, Sahakian BJ (2007a). A neuropsychological comparison of obsessive-compulsive disorder and trichotillomania. Neuropsychologia 45, 654-662.

Chamberlain SR, Fineberg NA, Menzies LA, Blackwell AD, Bullmore ET, Robbins TW, Sahakian BJ (2007b). Impaired cognitive flexibility and motor inhibition in unaffected first-degree relatives of patients with obsessivecompulsive disorder. American Journal of Psychiatry 164, 335-338.

Chamberlain SR, Menzies L, Hampshire A, Suckling J, Fineberg NA, del Campo N, Aitken M, Craig K, Owen AM, Bullmore ET, Robbins TW, Sahakian BJ (2008). Orbitofrontal dysfunction in patients with obsessive-compulsive disorder and their unaffected relatives. Science 321, 421-422.

Cools R, Roberts AC, Robbins TW (2008). Serotoninergic regulation of emotional and behavioural control processes. Trends in Cognitive Science 12, 31-40.

Crockett MJ, Clark L, Robbins TW (2009). Reconciling the role of serotonin in behavioral inhibition and aversion: acute tryptophan depletion abolishes punishment-induced inhibition in humans. Journal of Neuroscience 29, 11993-11999.

Cyders MA, Smith GT (2008). Emotion-based dispositions to rash action: positive and negative urgency. Psychological Bulletin 134, 807-828.

Endrass T, Kloft L, Kaufmann C, Kathmann N (2011). Approach and avoidance learning in obsessive-compulsive disorder. Depression and Anxiety 28, 166-172.

Endrass T, Schuermann B, Kaufmann C, Spielberg R, Kniesche R, Kathmann N (2010). Performance monitoring and error significance in patients with obsessivecompulsive disorder. Biological Psychology 84, 257-263.
Ersche KD, Roiser JP, Abbott S, Craig KJ, Muller U, Suckling J, Ooi C, Shabbir SS, Clark L, Sahakian BJ, Fineberg NA, Merlo-Pich EV, Robbins TW, Bullmore ET (2011). Response perseveration in stimulant dependence is associated with striatal dysfunction and can be ameliorated by a $\mathrm{d}(2 / 3)$ receptor agonist. Biological Psychiatry 70, 754-762.

Evers EA, van der Veen FM, van Deursen JA, Schmitt JA, Deutz NE, Jolles J (2006). The effect of acute tryptophan depletion on the BOLD response during performance monitoring and response inhibition in healthy male volunteers. Psychopharmacology (Berlin) 187, 200-208.

Figee M, Vink M, de Geus F, Vulink N, Veltman DJ, Westenberg H, Denys D (2011). Dysfunctional reward circuitry in obsessive-compulsive disorder. Biological Psychiatry 69, 867-874.

Foa EB, Huppert JD, Leiberg S, Langner R, Kichic R, Hajcak G, Salkovskis PM (2002). The ObsessiveCompulsive Inventory: development and validation of a short version. Psychological Assessment 14, 485-496.

Gehring WJ, Himle J, Nisenson LG (2000). Actionmonitoring dysfunction in obsessive-compulsive disorder. Psychological Science 11, 1-6.

Gillan CM, Papmeyer M, Morein-Zamir S, Sahakian BJ, Fineberg NA, Robbins TW, de Wit S (2011). Disruption in the balance between goal-directed behavior and habit learning in obsessive-compulsive disorder. American Journal of Psychiatry 168, 718-726.

Goodman WK, Price LH, Rasmussen SA, Mazure C, Fleischmann RL, Hill CL, Heninger GR, Charney DS (1989). The Yale-Brown Obsessive Compulsive Scale. I. Development, use, and reliability. Archives of General Psychiatry 46, 1006-1011.

Graybiel AM, Rauch SL (2000). Toward a neurobiology of obsessive-compulsive disorder. Neuron 28, 343-347.

Gu BM, Park JY, Kang DH, Lee SJ, Yoo SY, Jo HJ, Choi CH, Lee JM, Kwon JS (2008). Neural correlates of cognitive inflexibility during task-switching in obsessive-compulsive disorder. Brain 131, 155-164.

Henseler I, Gruber O, Kraft S, Krick C, Reith W, Falkai P (2008). Compensatory hyperactivations as markers of latent working memory dysfunctions in patients with obsessive-compulsive disorder: an fMRI study. Journal of Psychiatry and Neuroscience 33, 209-215.

Jung WH, Kang DH, Han JY, Jang JH, Gu BM, Choi JS, Jung MH, Choi CH, Kwon JS (2011). Aberrant ventral striatal responses during incentive processing in unmedicated patients with obsessive-compulsive disorder. Acta Psychiatrica Scandinavica 123, 376-386.

Kehagia A, Murray GK, Robbins TW (2010). Learning and cognitive flexibility: frontostriatal function and monoaminergic modulation. Current Opinion in Neurobiology 20, 199-204.

Koran LM, Hanna GL, Hollander E, Nestadt G, Simpson HB (2007). Practice guideline for the treatment of patients with obsessive-compulsive disorder. American Journal of Psychiatry 164 (Suppl. 7), 5-53.

Kuelz AK, Hohagen F, Voderholzer U (2004). Neuropsychological performance in obsessive- 
compulsive disorder: a critical review. Biological Psychology 65, 185-236.

Lipszyc J, Schachar R (2010). Inhibitory control and psychopathology: a meta-analysis of studies using the stop signal task. Journal of the International Neuropsychological Society 16, 1064-1076.

Maltby N, Tolin DF, Worhunsky P, O'Keefe TM, Kiehl KA (2005). Dysfunctional action monitoring hyperactivates frontal-striatal circuits in obsessive-compulsive disorder: an event-related fMRI study. Neuroimage 24, 495-503.

Menzies L, Achard S, Chamberlain SR, Fineberg N, Chen CH, del Campo N, Sahakian BJ, Robbins TW, Bullmore E (2007). Neurocognitive endophenotypes of obsessive-compulsive disorder. Brain 130, 3223-3236.

Menzies L, Chamberlain SR, Laird AR, Thelen SM, Sahakian BJ, Bullmore ET (2008). Integrating evidence from neuroimaging and neuropsychological studies of obsessive-compulsive disorder: the orbitofronto-striatal model revisited. Neurosciecne and Biobehavioural Reviews 32, 525-549.

Monterosso JR, Aron AR, Cordova X, Xu J, London ED (2005). Deficits in response inhibition associated with chronic methamphetamine abuse. Drug and Alcohol Dependence 79, 273-277.

Montgomery SA, Asberg M (1979). A new depression scale designed to be sensitive to change. British Journal of Psychiatry 134, 382-389.

Morein-Zamir S, Fineberg NA, Robbins TW, Sahakian BJ (2010). Inhibition of thoughts and actions in obsessivecompulsive disorder: extending the endophenotype? Psychological Medicine 40, 263-272.

Moritz S, Hubner M, Kluwe R (2004). Task switching and backward inhibition in obsessive-compulsive disorder. Journal of Clinical and Experimental Neuropsychology 26, 677-683.

Nelson HE (1982). The National Adult Reading Test Manual. NFER-Nelson: Windsor.

Nielen MM, den Boer JA, Smid HG (2009). Patients with obsessive-compulsive disorder are impaired in associative learning based on external feedback. Psychological Medicine 39, 1519-1526.

O'Doherty JP (2007). Lights, camembert, action! The role of human orbitofrontal cortex in encoding stimuli, rewards, and choices. Annals of the New York Academy of Sciences 1121, 254-272.

Olley A, Malhi G, Sachdev P (2007). Memory and executive functioning in obsessive-compulsive disorder: a selective review. Journal of Affective Disorders 104, 15-23.

Page LA, Rubia K, Deeley Q, Daly E, Toal F, Mataix-Cols D, Giampietro V, Schmitz N, Murphy DG (2009).
A functional magnetic resonance imaging study of inhibitory control in obsessive-compulsive disorder. Psychiatry Research 174, 202-209.

Penades R, Catalan R, Rubia K, Andres S, Salamero M, Gasto C (2007). Impaired response inhibition in obsessive compulsive disorder. European Psychiatry 22, 404-410.

Rauch SL, Wedig MM, Wright CI, Martis B, McMullin KG, Shin LM, Cannistraro PA, Wilhelm S (2007). Functional magnetic resonance imaging study of regional brain activation during implicit sequence learning in obsessive-compulsive disorder. Biological Psychiatry 61, 330-336.

Remijnse PL, Nielen MM, van Balkom AJ, Cath DC, van Oppen P, Uylings HB, Veltman DJ (2006). Reduced orbitofrontal-striatal activity on a reversal learning task in obsessive-compulsive disorder. Archives of General Psychiatry 63, 1225-1236.

Roth RM, Saykin AJ, Flashman LA, Pixley HS, West JD, Mamourian AC (2007). Event-related functional magnetic resonance imaging of response inhibition in obsessive-compulsive disorder. Biological Psychiatry 62, 901-909.

Sahakian BJ, Morein-Zamir S (2011). Depression and resilience: insights from cognitive, neuroimaging and psychopharmacological studies. In Decision Making, Affect, and Learning: Attention and Performance XXIII (ed. M. Delgado, E. Phelps and T. W. Robbins), pp. 505-532. Oxford University Press: Oxford

Spielberger CD, Gorsuch RL, Lushene R, Vagg PR, Jacobs GA (1983). Manual for the State-Trait Anxiety Inventory (Form Y). Consulting Psychologists Press: Palo Alto, CA.

Stahl SM (2008). Stahl's Essential Psychopharmacology: Neruoscientific Basis and Practical Applications. Cambridge University Press: New York.

Stern ER, Welsh RC, Fitzgerald KD, Gehring WJ, Lister JJ, Himle JA, Abelson JL, Taylor SF (2011). Hyperactive error responses and altered connectivity in ventromedial and frontoinsular cortices in obsessive-compulsive disorder. Biological Psychiatry 69, 583-591.

van den Heuvel OA, Veltman DJ, Groenewegen HJ, Cath DC, van Balkom AJ, van Hartskamp J, Barkhof F, van Dyck R (2005). Frontal-striatal dysfunction during planning in obsessive-compulsive disorder. Archives of General Psychiatry 62, 301-309.

Watkins LH, Sahakian BJ, Robertson MM, Veale DM, Rogers RD, Pickard KM, Aitken MR, Robbins TW (2005). Executive function in Tourette's syndrome and obsessive-compulsive disorder. Psychological Medicine 35, 571-582. 